\title{
The art of microscopy
}

This month, we celebrate the value and beauty of images of biological processes captured by microscopes.

$\mathrm{n}$ this November issue of Nature Methods, we continue our yearly tradition of featuring the Nikon 'Small World' Photomicrography Competition winner on our cover. This year's winning image of trichomes and stomata on a southern live oak leaf, captured by Jason Kirk of Baylor College of Medicine, certainly does not disappoint! It's one of our all-time favorites. Microscopes represent one of the few biological tools that are well known to non-scientists. As described in this month's Technology Feature, the growing trend of 'do-it-yourself' microscopy is helping bring imaging capabilities to a broader swath of people with inexpensive, simple devices made from materials such as paper. Researchers are also benefitting from this DIY trend: sophisticated instruments can be built from modular components, which, when coupled with open-source software, facilitate cutting-edge imaging analyses.

Striking images, whether captured by a microscope or created (such as that of the SARS-CoV-2 virus particle), serve as a powerful tool for communication, one that scientists should more often take advantage of for explaining concepts and knowledge to the broader public. Our cover image, along with the other top 20, honorable mentions and 'images of distinction' featured on this page, are perfect examples of how the beauty of tiny creatures and close-up views of biological processes can capture the rapt attention of scientists and non-scientists alike.

Published online: 3 November 2021

https://doi.org/10.1038/s41592-021-01324-y

A selection of Small World contest images.

Clockwise, from top left: damselfly larva, image of distinction, captured by Robert C. Berdan; green monkey cells, image of distinction, captured by

Christophe Leterrier and Ghislaine Caillol; sensory neuron from an embryonic rat, 4th place, captured by Paula Diaz; mouse intestine cross-section, 8th place, captured by Amy Engevik; mold on a cherry stem, honorable mention, captured by Sergii Dymchenko; mouse mammary gland ductal tree, image of distinction, captured by Sounak

Sahu; human skin organoid, image of distinction, captured by Jiyoon Lee and Karl R. Koehler; Drosophila ovaries, image of distinction, captured by Wen Lu and Vladimir I. Gelfand; breast organoid, 12th place, captured by Jakub Sumbal; army cutworm moth abdomen cross-section, image of distinction, captured by James Miess.
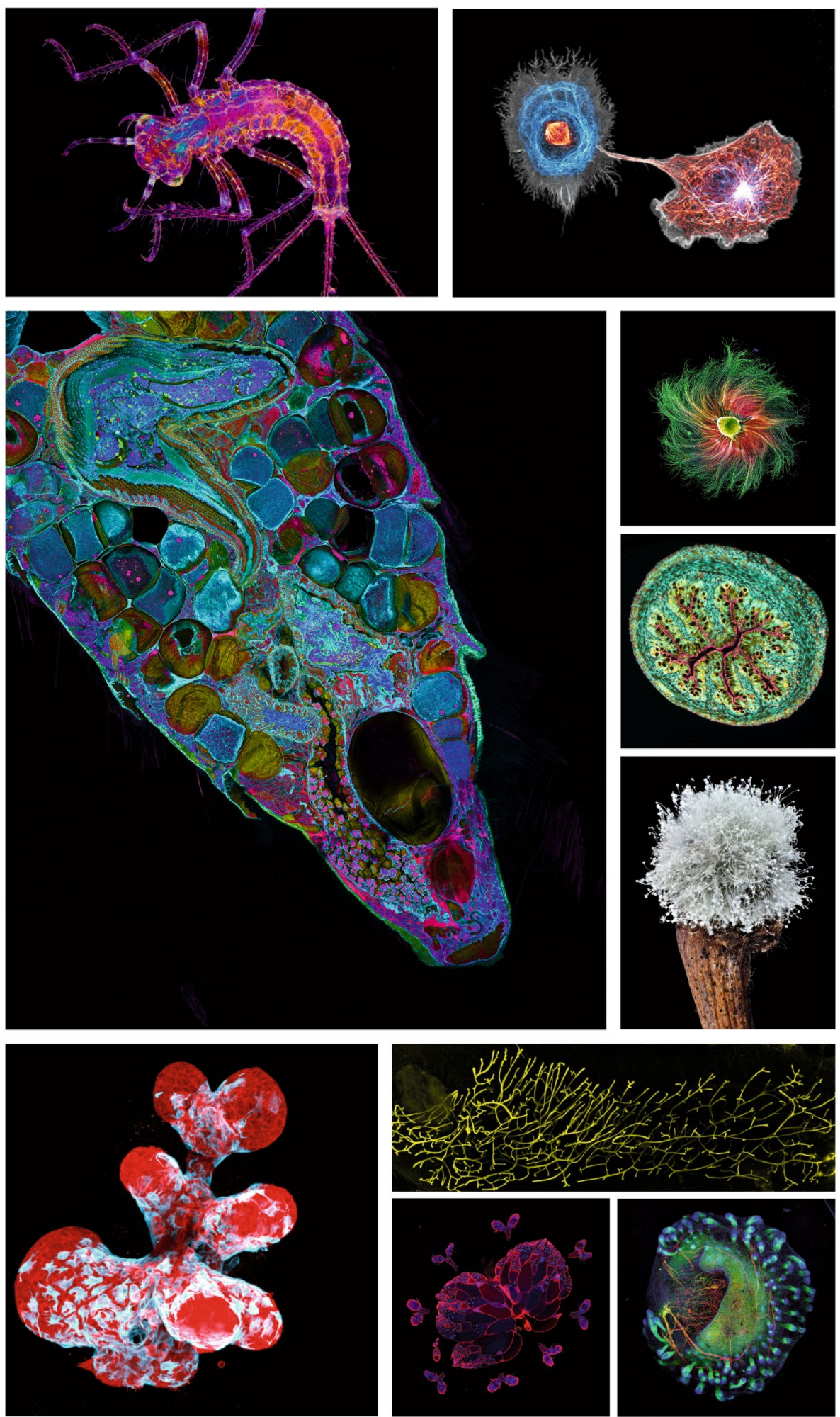